\title{
Bunsetsu Identification Using Category-Exclusive Rules
}

\author{
Masaki Murata Kiyotaka Uchimoto Qing Ma Hitoshi Isahara \\ Communications Research Taboratory, Ministry of Posts and Telecommunications \\ 588-2, Jwaoka, Nishi-ku, Kobe, 651-2492, Japan \\ tel:-81-78-969-2181 fax:+81-78-969-2189 http://www-karc.crl.go.jp/ips/murata \\ \{murata,uchimoto,qma,isahara\}@crl.go.jp
}

\begin{abstract}
This paper describes two now bunsetsu identification methods using supervised loaming. Since Japanese syntactic analysis is usually done after bunsetsu identification, bunsetsu identification is important for analyzing Japanese sentences. In cxperiments comparing the four previously available machinelearning methods (decision tree, maximum-entropy method, example-based approach and docision list) and two now mothods using category-exclusive rules, the new mothod using the category-exchusive rules with the highest similarity performed bost.
\end{abstract}

\section{Introduction}

This paper is abont machine Iearning methods for idcntifying bunsetsus, which correspond to English phrasal units such as noun phrases and prepositional phrases. Since Japanese syntactic analysis is usually done after bunsetsu identification (Uchinoto et al., 1999), identifying bunsetsu is important for analyring Japaneso sentences. 'The conventional studies on bunsetsu identification ${ }^{1}$ have used hand-made rules (Kameda, J995; Kurohashi, 1.998), but bunsetsu identification is not an easy task. Conventional studies used many hand-made rules developed at the cost of many man-hours. Kurohashi, for example, made 146 rules for bunsetsu iclentification (Kurohashi, 1998).

In an attempt to reduce the nunnber of manhours, wo used machine-learning methods for bunsetsu identification. Because it was not clear which machine-learning method would be the one most appropriate for bunsetsu identification, so we tried a variety of them. In this paper we report experimonts comparing four machine-leaming methods (decision tree, maximum entropy, example-based, and decision list nethods) and our now mothods using category-exclusive rules.

\footnotetext{
TBunsetsu identilication is a problem similar to chunking (Rannshaw and Marcus, 1995; Sang and Veonstra, 1999) in other languages.
}

\section{Bunsetsu identification problem}

We conducted experiments on the following supervised learning methods for identifying bunsetsu:

- Decision tree method

- Maxinum entropy mothod

- Example-based method (use of similarity)

- Decision list (use of probability and frequency)

- Mothod 1 (usse of exchusive rules)

- Method 2 (use of exchusive rules with the highest similarity).

In gencral, bunsetsu identification is dono after morphological and before syntactic analysis. Morphological analysis corresponds to part-of-specch tagging in English. Japanese syntactic structures are usually represented by the relations between bumsotsus, which comespond to phrasal units such as a nom phraso or a prepositional phrase in English. So, bunsetsu identification is inportant in Japanese sentence analysis.

In this paper, we identify a bunsetsu by using information from a morphological analysis. Bunsetsu identification is treated as the task of deciding whether to insert a "l" mark to indicate the partition between two bunsetsus as in Figure 1. Therefore, bunsetsu identification is done by judging whether a partition mark should be inserted between two adjacent morphomes or not. (Wo do not use the inserted partition mark in the following analysis in this paper for the sake of simplicity.)

Our bunsetsu identification method uses the morphological information of the two preceding and two succeding morphemes of an analyzed space between two adjacent morphemes. Wo use the following mor'phological information:

(i) Major part-of-speech (POS) category, ${ }^{2}$

(ii) Minor POS category or inflection type,

(iii) Somantic information (the first threo-digit number of a category number as used in "BGI" (NI.RI, 1964)),

${ }^{2}$ Jart-ol-spech categories follow those of JUMAN (Kunolashli and Nagao, 1998). 


\begin{tabular}{l|ll|l} 
boku $\quad \begin{array}{l}\text { ga } \\
\text { (I) nominative-case particle }\end{array}$ & $\begin{array}{l}\text { bunsetsu } \\
\text { (bunsetsu) }\end{array}$ & wo & objective-case particle
\end{tabular} \mid $\begin{aligned} & \text { matomeageru } \\
& \text { (identify) }\end{aligned}$.

Figure 1: Example of identified bunsetsus

\begin{tabular}{|lllll|} 
& $\begin{array}{l}\text { bun } \\
\text { (sentence) }\end{array}$ & $\begin{array}{l}\text { wo } \\
\text { (obj) }\end{array}$ & $\begin{array}{l}\text { kugiru } \\
\text { (divide) }\end{array}$ & $\cdot$ \\
Major POS & Noun divide sentences) & Particle & Verb & Symbol \\
Minor POS & Normal Noun & Case-Particle & Normal Form & Punctuation \\
Semantics & $\times$ & None & 217 & $\times$ \\
Word & $\times$ & wo & kugiru & $\times$ \\
\hline
\end{tabular}

Figure 2: Information used in bunsetsu identification

(iv) Word (Iexical information).

For simplicity we do not use the "Semantic information" and "Word" in either of the two outside morphemes.

Figure 2 shows the information used to judge whether or not to insert a partition mark in the space between two adjacent morphemes, "wo (obj)" and "kugiru (divide)," in the sentence "bun wo kugiru. ((I) divide sentences)."

\section{Bunsetsu identification process for each machine-learning method}

\subsection{Decision-tree method}

In this work we used the program C4.5 (Quinlan, 1995) for the decision-tree lcarning method. The four types of information, (i) major POS, (ii) minor POS, (iii) semantic information, and (iv) word, mentioned in the previous section were also used as features with the decision-tree learning method. As shown in Figure 3, the number of features is 12 $(2+4+4+2)$ because we clo not use (iii) scmantic information and (iv) word information from the two outside morphemes.

In Figure 2, for example, the value of the feature 'the major POS of the far left morpheme' is 'Noun.'

\subsection{Maximum-entropy method}

The maximum-entropy method is useful with sparse data conditions and has been used by many researchers (Berger et al., 1996; Ratnaparkhi, 1996; Ratnaparkhi, 1997; Borthwick et al., 1998; Uchimoto et al., 1999). In our maximum-entropy experiment we used Ristad's system (Ristad, 1998). The analysis is performed by calculating the probability of inserting or not inserting a partition mark, from the output of the system. Whichever probability is higher is selected as the desired answer.
In the maximum-entropy method, we use the same four types of morphological information, (i) major POS, (ii) minor POS, (iii) semantic information, and (iv) word, as in the decision-tree method. However, it does not consider a combination of features. Unlike the decision-tree method, as a result wo had to combine features manually.

First we considered a combination of the bits of each morphological information. Because thore were four types of information, the total number of combinations was $2^{4}-1$. Since this number is large and intractable, we considered that (i) major POS, (ii) minor POS, (iii) semantic information, and (iv) word information gradually become more specific in this order, and wo combined the four types of information in the following way:

\footnotetext{
Information A: (i) major POS

Information B: (i) major POS and (ii) minor POS

Information C: (i) major POS, (ii) minor POS and

(iii) semantic information

Information D: (i) major POS, (ii) minor POS,
}

(iii) semantic information and (iv) word

We used only Information A and B for the two outside morphemes because we did not use semantic and word information in the same way it is used in the decision-tree method.

Next, we considered the combinations of cach type of information. As shown in Figure 4, the number of combinations was $64(2 \times 4 \times 4 \times 2)$.

For data sparseness, in addition to the above combinations, we considered the cases in which first, one of the two outside morphemes was not used, secondly, neither of the two outside ones worc used, and thirdly, only one of the two middle ones is used. The number of features used in the maximum-cntropy method is 152 , which is obtained as follows: ${ }^{3}$

\footnotetext{
${ }^{3}$ When we extracted features from all of the articles on
} 


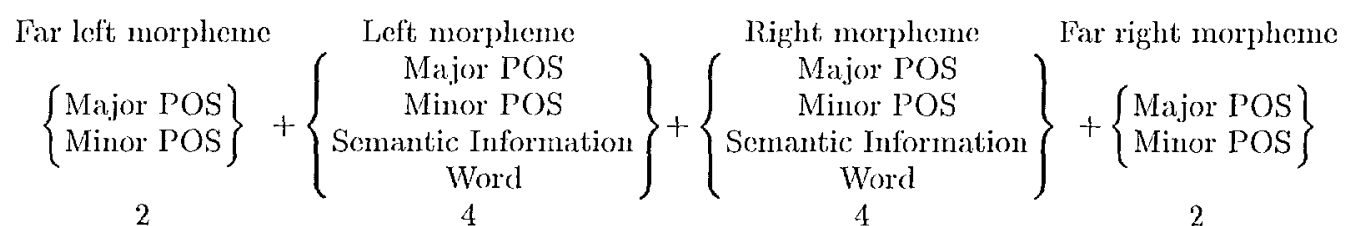

2

2

Figure 3: Features used in the decision-tree mothod

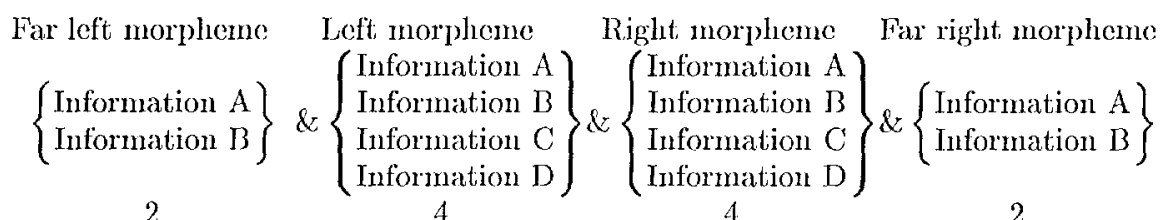

4

2

Figure 4: Features used in the maximum-cntropy method.

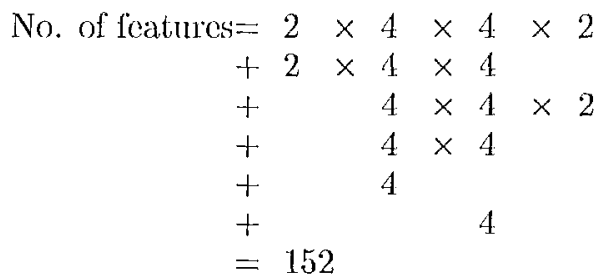

In Figure 2, the feature that uses Information $B$ in the far left morpheme, Information D in the left morphene, Information $\mathrm{C}$ in the right morpheme, and Information $A$ in the fal right nuorpheme is "Nonn: Normal Nomr; Particle: CaseParticle: none: wo; Verb: Normal Form: 217; Symbol". In the maximum-entropy mothod we used for each space 152 features such as this one.

\subsection{Example-based method (use of similarity)}

An example-based method was proposed by Nagao (Nagao, 1984) in an attempt to solve problems in machine translation. To resolve a problem, it uses the most similar example. In the present, work, the example-based mothod impartially used the same four types of information (sce Eq. (1)) as used in the maximum-entropy method.

To use this method, we must define the similarity of an input to an example. We use the 152 patiterns from the maximum-entropy method to establish the level of similarity. We define the similarity $S$ between an input and an cxample according to which one of these 152 levels is the matching level, as follows. (The equation reflects the importance of the two middle morphemes.)

Jannary 1, 1995 of a Kyoto University corpus (the number of spaces between morpliemes was 25,814 ) by using this method, the number of ty]es of features was $1,534,701$.

$$
\begin{aligned}
& \mathrm{S}=s\left(m_{-1}\right) \times s\left(m_{+-1}\right) \times 10,000 \\
& \quad+s\left(m_{-2}\right) \times s\left(m_{+-2}\right)
\end{aligned}
$$

Here $m_{-1}, m_{+-1}, m_{-2}$, and $m_{+2}$ refer respectively to the left, right, far left, and far right, morphemes, and $\mathrm{s}(\mathrm{x})$ is the morphological similarity of a morpheme $x$, which is defined as follows:

$$
\begin{aligned}
s(x)= & 1 \text { (when no information of } x \text { is matched) } \\
& 2 \text { (when Information A of } x \text { is matched) } \\
& 3 \text { (when Information } B \text { of } x \text { is matehed) } \\
& 4 \text { (when Information } C \text { of } x \text { is matched) } \\
& 5 \text { (when Information } D \text { of } x \text { is matched) }
\end{aligned}
$$

Figure 5 shows an example of the levels of similarity. When a pattern matches Information A of all four morphemes, such as "Noun; Particle; Verb; Symbol", its similarity is 40,004 $(2 \times 2 \times 1.0,000+$ $2 \times 2$ ). When a pattom matches a pattern, such as "-.; Particle: Case-Particle: none: wo;--; --", its similarity is $50,001(5 \times 1 \times 10,000+1 \times 1)$.

The example-based mothod extracts the example with the highest level of similarity and checks whether or not that example is marked. A partition mark is inserted in the input data only when the example is marked. When multiple examples have the same highest level of similarity, the selection of the best example is ambiguous. In this case, we count the number of marked and ummarked spaces in all of the examples and choose the larger.

\subsection{Decision-list method (use of probability and frequency)}

The decision-list method was proposed by Rivest (Rivest, 1987), in which the rules are not expressed as a tree structure like in the decision-tiec method, 


\begin{tabular}{|lcllll|} 
& & bun & wo & kugiru &. \\
& $\mathrm{s}(\mathrm{x})$ & $\begin{array}{l}\text { (sentence) } \\
m_{-2}\end{array}$ & $\begin{array}{l}\text { (obj) } \\
m_{-1}\end{array}$ & $\begin{array}{l}\text { (divide) } \\
m_{+1}\end{array}$ & $m_{+2}$ \\
No information & 1 & - & -- & -- & - \\
Information A & 2 & Noun & Particle & Verb & Symbol \\
Information B & 3 & Normal Noun & Casc-Particle & Normal Form & Punctuation \\
Information C & 4 & $\times$ & None & 217 & $\times$ \\
Information D & 5 & $\times$ & wo & kugiru & $\times$ \\
\hline
\end{tabular}

Figure 5: Example of levels of similarity

but are expanded by combining all the features, and are stored in a one-dimensional list. A priority order is defined in a certain way and all of the rules are arranged in this order. The decision-list mothod searches for rules from the top of the list and analyzes a particular problem by using only the first applicable rule.

In this study we used in the decision-list method the same 152 types of patterns that were used in the maximum-entropy method.

To determine the priority order of the rules, we referred to Yarowsky's mothod (Yarowsky, 1994) and Nishiokayama's method (Nishiokayama ct al., 1998) and used the probability and frequency of cach rule as measures of this priority order. When multiple rules had the same probability, the rules were arranged in order of their frequency.

Suppose, for cxample, that Pattcrn A "Noun: Normal Noun; Particle: Case-Particle: none: wo; Verb: Normal Form; 217; Symbol: Punctuation" occurs 13 times in a learning set and that ten of the occurrences include the inserted partition mark. Suppose also that Pattern B "Noun; Particle; Verb; Symbol" occurs 123 times in a learning set and that, 90 of the occurrences include the mark.

This example is recognized by the following rules:

Pattern $A \Rightarrow$ Partition $76.9 \%(10 / 13)$, Freq. 23

Pattcrn B $\Rightarrow$ Partition $73.2 \%(90 / 123)$, Freq. 123

Many similar rules woro made and were then listed in order of their probabilities and, for any one probability, in order of their frequencies. This list was searched from the top and the answer was obtained by using the first applicable rule.

\subsection{Method 1 (use of category-exclusive rules)}

So far, we have described the four existing machine learning methods. In the next two sections we describe our methods.

It is reasonable to consider the 152 patterns used in three of the previous methods. Now, let us suppose that the 152 patterns from the learning set yield the statistics of Figure 6 .
"Partition" means that the rule determines that a partition mark should be inserted in the input data and "non-partition" means that the rule detcrmines that a partition mark should not be inserted.

Suppose that when we solve a hypothetical problem Paticrns A to $\mathrm{G}$ are applicable. If we use the decision-list method, only Rule A is used, which is applicd first, and this determines that a partition mark should not be inserted. For Rules B, C, and $D$, although the frequency of cach rule is lower than that of Rule A, the sum of their frequencies of the rules is higher, so we think that it is better to use Rules B, C, and D than Rule A. Mothod 1 follows this idea, but we do not simply sum up the frequencics. Instead, we count the number of examples used in Rules B, C, and D and judge the category having: the largest number of examples that satisfy the pattern with the highest probability to be the dosired answer.

For example, suppose that in the above example the number of examples satisfying Rules B, C, and $\mathrm{D}$ is 65 . (Because some examples overlap) in multiple rules, the total number of examples is actually smaller than the total number of the fropuencies of the three rules.) In this case, among the examples used by the rules having $100 \%$ probability, the number of examples of partition is 65 , and the number of examples of non-partition is 34 . So, we deternine that the desired answer is to partition.

A rule having $100 \%$ probability is called a category-exclusive rulc because all the data satisfying it belong to one category, which is either partition or non-partition. Because for any given space the number of rules used can be as large as 152 , category-exclusive rules are applied often ${ }^{4}$. Method 1 uses all of these catcgory-cxclusive rules, so we call it the method using category-exclusive rules.

Solving problems by using rules whose probabilities are not $100 \%$ may result in the wrong solutions. Almost all of the traditional machine learning methods solve problems by using rules whose probabilities

\footnotetext{
'The ratio of the spaces analyzed by using categoryexclusive rules is $99.30 \%(16864 / 16983)$ in Experiment 1 of Section 4. This indicates that almost all of the spaces are analyzed by category-exclusive rules.
} 


\begin{tabular}{|c|c|c|c|c|c|}
\hline Runle A: & l'attern $A$ & $\Rightarrow$ & probability of nom-partition & $100 \%(34 / 34)$ & Frecuency 34 \\
\hline Rule $\mathrm{B}$ : & Patteru 13 & $\Rightarrow$ & probability of partition & $100 \%(33 / 33)$ & Frequency 33 \\
\hline Jule $C$ : & Pattern C & $\Rightarrow$ & probability of partition & $100 \%(25 / 25)$ & Freculuency 25 \\
\hline Rule D: & Pattern I) & $\Rightarrow$ & probability of partition & $100 \%(19 / 19)$ & Frecuency 19 \\
\hline Rule E: & Pattern ] & $\Rightarrow$ & probability of partition & $81.3 \%(100 / 123)$ & Frequency 123 \\
\hline Rule F: & Pattorn F & $\Rightarrow$ & probability of partition & $76.9 \%(10 / 13)$ & Frequency 13 \\
\hline Rivle G: & Pattern G & $\Rightarrow$ & probability of non-partition & $57.4 \%(310 / 540)$ & Frecutency 540 \\
\hline
\end{tabular}

ligme 6: an example of rules used in Method 1

are not 100\%. By using such methods, we cannot, hope to improve accuracy. If we want to improve accuracy, we must use category-oxchusive rules. There are some cases, however, for which, cven if we take this approach, category-exelusive rules are rarely alpplied. In such (ases, wo must adel now fealiures to the analysis to croato a situation in which many category-exclusive rules an be applierl.

However, it is not, suflicient to nse categoryexchusive rules. There are many modningless rules which happen to be catogory-exchusive only in a learning set. Wo nust consider how to eliminate such moaningless rules.

\subsection{Method 2 (using category-exclusive rules with the highest similarity)}

Method 2 combines the eximplo-based method and Method 1. That is, it combines the method using similarity and the method using category-xehsive rules in order to eliminate the meaningless categoryexchusive rules mentioned in the provious section.

Mothod 2 also mses 152 pations for identilying bunsotsm. These patterns ane used as rules in the sanne way as in Method 1. Jesired answels are determined by using the rule having the highest probalility. When multiple rules have the same probability, Method 2 uses the value of the similarity described in the section of the examplo-beased method and analyzes the problem with the mle having the highest similarity. When multiple rules have the same probability and similarity, the nothod tiakes the examples used by the rules having the highest probability and the highest similarity, and chooses the category with the larger number of examples as the desired answer, in the sane way as in Method 1.

However, when ategory-exclusive rules having more than one firequency exist, the above procedure is performed after eliminating all of the categoryexclusive rules having one frequency. In other words, category-exchusive rules having more than one fiequency are given a higher priority than categoryexclusivo rules having only one froduency but having a higher similarity. This is because catcogoryexchusive rules having only one frequency are not, so roliable.

\section{Experiments and discussion}

In our experiments we used a Kyoto University toxt corpus (Kurohashi and Nagao, 1997), which is a tagged corpus made up of articles from the Mainichi newspaper. All experitnents reported in this paper were performed using articles dated fiom Jannary 1 to 5, 1995. Wo obtained the correct information on morphology and bunsetsu irlentification fiom the tagged corpus.

The following experiments wore conducted to determine which supervised learning mothod achioxes the highest acenuacy rate.

- Experiment 1

Learning; set: January 1, 1995

Test sct: Jamuaty 3, 1995

- Experiment 2

Toaming sot: Jammary 1, 1995

'Test set: Jamuary 5, 1995

Becanse we nsed Tixperimont 1 in making Mothod 1. and Method 2, Experiment 1 is a closed data set for Method 1 and Method 2. So, we performed bxperinent, 2.

The results are listed in 'lables 1 to 4. We used KNI2.0b4 (Kurohashi, 1997) and KNP2.(1)6 (Kurohashi, 1998), which are bunsetsu identification and syntactic analysis systems using many hand-nade rules in addition to the six methods clescribed in Section 3. Because KNP is not based on a machine learning mothod but many hand-nado rules, in the KNI' results "Lcarning set" and "Test set" in the talbles have no meanings. In the experiment of KNP, we also uses morphological information in a corpus. The "F" in the tables indicates the $\mathrm{F}$-measure, which is the hammonic mean of a recall and a precision. A recall is the fraction of correctly identified partitions out of all the partitions. A precision is the fraction of correctly identified partitions ont of all the spaces which were judged to have a partition mark inserted.

'ables 1. to 4 show the following results:

- In the test set the clecision-tree method was a litite better than the maximum-cntropy 
Table 1: Results of learning set of Experiment 1

\begin{tabular}{|l|r|r|r|}
\hline \multicolumn{1}{|c|}{ Method } & \multicolumn{1}{c|}{$\mathrm{F}$} & \multicolumn{1}{c|}{ Recall } & Precision \\
\hline Decision Tree & $99.58 \%$ & $99.66 \%$ & $99.51 \%$ \\
Maximum Entropy & $99.20 \%$ & $99.35 \%$ & $99.06 \%$ \\
Example-Based & $99.98 \%$ & $100.00 \%$ & $99.97 \%$ \\
Decision List & $99.98 \%$ & $100.00 \%$ & $99.97 \%$ \\
Method 1 & $99.98 \%$ & $100.00 \%$ & $99.97 \%$ \\
Method 2 & $99.98 \%$ & $100.00 \%$ & $99.97 \%$ \\
\hline KNP 2.0b4 & $99.23 \%$ & $99.78 \%$ & $98.69 \%$ \\
KNP 2.0b6 & $99.73 \%$ & $99.77 \%$ & $99.69 \%$ \\
\hline
\end{tabular}

The number of spaces between two morphemes is 25,814 . The number of partitions is 9,523 .

Table 2: Results of test set of Experiment 1

\begin{tabular}{|l|c|c|r|}
\hline \multicolumn{1}{|c|}{ Method } & $\overline{1}$ & Recall & Precision \\
\hline Decision Tree & $98.87 \%$ & $98.67 \%$ & $99.08 \%$ \\
Maximum Entropy & $98.90 \%$ & $98.75 \%$ & $99.06 \%$ \\
Examplc-Based & $99.02 \%$ & $98.69 \%$ & $99.36 \%$ \\
Decision List & $98.95 \%$ & $98.43 \%$ & $99.48 \%$ \\
Method 1 & $98.98 \%$ & $98.54 \%$ & $99.43 \%$ \\
Method 2 & $99.16 \%$ & $98.88 \%$ & $99.45 \%$ \\
\hline KNP 2.0b4 & $99.13 \%$ & $99.72 \%$ & $98.54 \%$ \\
KNP 2.0b6 & $99.66 \%$ & $99.68 \%$ & $99.64 \%$ \\
\hline
\end{tabular}

The number of spaces between two morphemes is 16,983 . The number of partitions is 6,166 .

method. Although the maximum-entropy method has a weak point in that it does not lcarn the combinations of fcatures, we could overcome this weakness by making almost all of the combinations of features to produce a higher: accuracy rate.

- The decision-list method was better than the maximum-entropy method in this experiment.

- The example-based method obtained the highest accuracy rate among the four existing methods.

- Although Method 1, which uses the categoryexclusive rule, was worse than the examplebased method, it was better than the decisionlist method. One reason for this was that the decision-list method chooses rules randomly when multiple rules have identical probabilities and frequencies.

- Method 2, which uses the category-exclusive rule with the highest similarity, achicved the highest accuracy rate among the supervised learning methods.

- The example-based method, the decision-list method, Method 1 and Method 2 obtained accuracy rates of about $100 \%$ for the learning set. This indicates that these methods are especially
Table 3: Results of learning set of Experiment 2

\begin{tabular}{|l|c|r|r|}
\hline \multicolumn{1}{|c|}{ Method } & \multicolumn{1}{c|}{$\mathrm{F}$} & \multicolumn{1}{c|}{ Recall } & Precision \\
\hline Decision Tree & $99.70 \%$ & $99.71 \%$ & $99.69 \%$ \\
Maximum Entropy & $99.07 \%$ & $99.23 \%$ & $98.92 \%$ \\
Example-Based & $99.99 \%$ & $100.00 \%$ & $99.98 \%$ \\
Decision List & $99.99 \%$ & $100.00 \%$ & $99.98 \%$ \\
Method 1 & $99.99 \%$ & $100.00 \%$ & $99.98 \%$ \\
Method 2 & $99.99 \%$ & $100.00 \%$ & $99.98 \%$ \\
\hline KNP 2.0b4 & $98.94 \%$ & $99.50 \%$ & $98.39 \%$ \\
KNP 2.0b6 & $99.47 \%$ & $99.47 \%$ & $99.48 \%$ \\
\hline
\end{tabular}

The number of spaces between two morphemes is 27,665 . The number of partitions is 10,143 .

Table 4: Results of test set of Experiment 2

\begin{tabular}{|l|c|c|r|}
\hline \multicolumn{1}{|c|}{ Method } & $\overline{\mathrm{F}}$ & $\overline{\text { Recall }}$ & Precision \\
\hline Decision Tree & $98.50 \%$ & $98.51 \%$ & $98.49 \%$ \\
Maximum Entropy & $98.57 \%$ & $98.55 \%$ & $98.59 \%$ \\
Example-Based & $98.82 \%$ & $98.71 \%$ & $98.93 \%$ \\
Decision List & $98.75 \%$ & $98.27 \%$ & $99.23 \%$ \\
Method 1 & $98.79 \%$ & $98.54 \%$ & $99.43 \%$ \\
Method 2 & $98.90 \%$ & $98.65 \%$ & $99.15 \%$ \\
\hline KNP 2.0b4 & $99.07 \%$ & $99.43 \%$ & $98.71 \%$ \\
KNP 2.0b6 & $99.51 \%$ & $99.40 \%$ & $99.61 \%$ \\
\hline
\end{tabular}

The number of spaces between two morphemes is 32,304 . The number of partitions is 11,756 .

strong for learning sets.

- The two methods using similarity (cxamplebased method and Method 2) were always better than the other methods, indicating that the use of similarity is effective if we can define it; appropriately.

- Wo carricd out experiments by using KNP, a system that uses many hand-made rules. The F-measure of KNP was highest in the test set.

- Wo used two versions of KNP, KNP $2.0 \mathrm{~b} 4$ and KNP 2.0b6. The latter was much better than the former, indicating that the improvements made by hand are effective. But, the maintenance of rules by hand has a limit, so the improvements made by hand are not always effective.

The above experiments indicate that Method 2 is best among the machine learning methods ${ }^{5}$.

In Table 5 we show some cases which were partitioned incorrectly with KNP but correctly with

\footnotetext{
${ }^{5}$ In these experiments, the differences were very small. But, we think that the differences are significant to some extent because we performed Experiment 1 and Experiment 2 , the data we used are a large corpus containing about a fow ten thousand morphemes and 1agged objectively in advance, and the difference of about $0.1 \%$ is large in the precisions of $99 \%$,
} 
Table 5: Cases when KNP was incortect and Method 2 was comect

\begin{tabular}{|c|c|}
\hline $\begin{array}{ll}\text { kotsukotsu } & \text { gaman-shi } \\
\text { (steadily) } & \text { (be pationt with) } \\
\text { (... be paticnt with ... steadily) }\end{array}$ & \\
\hline $\begin{array}{l}\text { yoyuu wo } \mid \text { motte }\left.\right|^{\text {Neno }} \\
\text { (cuough strength) obj (have) } \\
\text { (... beat off ... having enough streng }\end{array}$ & $\begin{array}{l}\text { shirizoke } \\
\text { (beat off) } \\
\text { gith) }\end{array}$ \\
\hline $\begin{array}{l}\text { Faisha wo | gurupu-uake } \\
\text { compand obj (grouping) } \\
\text { (... do grouping companies) }\end{array}$ & $\begin{array}{l}\text { shite } \\
(\mathrm{do})\end{array}$ \\
\hline
\end{tabular}

Method 2. A partition with "NEED" indicates that KNP missed inserting the partition mark, and a partition with "WRONG" indicates that $\mathrm{KNI}$ " inserted the partition mark incorrectly. In the test set of Experiment 1, the F-moasure of KNP2.0b6 was $99.66 \%$. The F-measure increases to $99.83 \%$, under the assumption that when KNP2.0b6 or Method 2 is correct, the answer is correct. Although the anceuracy rate for KNP2.0b6 was high, thero were some cases in which KNP partitioned incomectly and Method 2 partitioned correctly. A combination of Mothod 2 with KNP2.0b6 may be able to improve the Fmeasure.

The only previous rescarch resolving bunsetsu identification by machine learning mothods, is the work by Zhang (Zhang and Ozeki, 1998). The, decision-tree mothod was used in this work. But this work used only a small number of information for bunsetsu identification ${ }^{i}$ and did not, achicve high accuracy latios. (The recall mate was $97.6 \%(=2502 /(2502+62))$, the precision rate, was $92.4 \%(=2502 /(2502+205))$, and $F$-measure was $94.2 \%$.

\section{Conclusion}

To solve the problem of accurate bunsetsu identification, we carried out experiments comparing four cxisting machine-learning methods (decisiontree method, maximum-entropy method, examplebased method and decision-list method). We obtained the following order of accuracy in bunsetsu identification.

\section{Example-Based $>$ Decision List $>$ Maximum Entropy $>$ Decision Thee}

We also described a now method which uses category-exclusive rules with the highest similarity. This method performed better than the other learning methods in our experiments.

\footnotetext{
Grihis work used only the l'OS information of the two morphemes of an analyzed space.
}

\section{References}

Ndam I. Bergor, Stephon A. Della Jietra, and Vincent J. Jella Pietial. 1996. A Maximum Entropy Approich to Natural Janguage Processing. Computational Linguistics, $22(1): 39-71$.

Andrew 33orthwick, John Sterling, Eugene Agichtejn, and Ralph Grishman. 1998. Exploiting Diverse Knowledge Sources via Maxinum Entropy in Named Entity lkecognition. In Proceedings of the Sirth Workshop on Very Large Corpore, pages 152160 .

Masayuki Kameda. 1995. Simple Japanese analysis tool q-jp. The Association for Natural Language l'rocessing, the tst National Convention, pages 349-352. (in Japanese).

Sadao Kurohashi and Makoto Nagao. 1997. Kyoto University text corpus project. pages 115-118. (in Japanese).

Sadao Kurolıashi and Makoto Nagao, 1998. Japanese Morphological Analysis System JUMAN version 3.5. J)epartment of Informatics, Kyoto University. (in Japanese).

Sadao Kurohashi, 1997. Japanese Dependency/Case Structure Analyzer KNI version 2.0b\%. Department of Informatics, Kyoto University. (in Jajanese).

Sadao Kurohashi, 1998. Japanese Dependency/Case Structure Analyzer KNI version 2.Ob6. Department of Informatics, Kyoto University. (in Japanese).

Makoto Nagao. 1981. A Famework of a Mechanical 'Translation between Japanese and Inglish by Analogy Principle. Artificial and Iuman lutelligence, pages 173-180.

Shigeyuki Nishokayana, 'Takehito Utsuro, and Yuji Matsmmoto. 1998. Fixtracting preference of dependency betwoen Tapanese subordinate clauses from corpus. $I S I C H-$ WGNJC C98-11, pages 31-38. (in .Japnese).

NIRI. 1964. (National Language liesearch Institute). Word List by Semantic Principles. Syuci Syuppan. (in Japanese).

J. R. Quinlan. 1995. Programs for machine learning.

lance A. Ramshaw and Mitchel! P. Marcus. 1995. Text clunking using transformation-based learning. In Procecdings of the Thivel Workshop on Very Large Corpora, pages 82.94.

Adwait Tatmaparkli. 1996. A Maximum Jutropy Model for Part-of-Speed 'lagging. Procedings of t'mpirical Method for Natural Language Processings, pages 133-142.

Adwait Ratmaparkli. 1997. A I inear Observed lime Statistical Jarser Based on Maximum Entropy Morlels. In Irocodings of Empirical Method for Natural Language I'rocessings.

Gic Sven listial. 1998. Maximum Entropy Modeling Ioolkit, Release 1.6 beta. http://www.mmemonic.com/ software/memt.

Ronald I. Rivest. 1987. I,carning Decision Iists. Machine Learning, 2:229-246.

Wrik F. Tjong Kim Sang and Jorn Veenstral. 1999. Representing text chunks. In $L A C L, 99$.

Kiyotaka Uchimoto, Satoshi Sekine, and Hitoshi Isahara. 1999. Japanese dependency structure analysis based on maximum entropy models. In Procedings of the Ninth Conference of the Suropcan Chapter of the Association for Computational Linguistics (BACL), pages 196-203.

David Yarowsky. 1994. Decision lists for lexical ambiguity resolution: $\Lambda$ pplication to accent restoration in Spanish and French. In 32th Annual Mceting of the Associtation of the Computational Linguistics, pages 88-95.

Yujic Khang and Kazuhiko Ozeki. 1998. 'The application of classification trees to bunsetsu segmentation of Japanese sentences. Journal of Natural Language Processing, 5(4):1.7-33. 